\title{
Indicadores e fatores de risco da cárie dentária em crianças no Brasil - uma revisão de literatura
}

\author{
Indicators and risk factors of dental caries in children in Brazil \\ - a literature review
}

Lenita Marangoni Lopes*

Fabiana Lima Vazquez ${ }^{* *}$

Antônio Carlos Pereira**

Dayse Andrade Romão ${ }^{* * * *}$

\section{Resumo}

Atualmente, a prevalência de cárie dentária encontra-se localizada em uma parcela da população, o que caracteriza sua polarização. A identificação desses grupos de risco permite que medidas preventivas, tanto comunitárias quanto individuais, sejam voltadas aos indivíduos desses grupos, resultando num uso mais eficiente dos recursos. Objetivo: este estudo objetiva verificar os fatores e preditores do risco da cárie estudados no Brasil, através de uma revisão da literatura. Materiais e método: Realizou-se uma busca na base de dados PUBMED utilizando-se os termos dental caries, risk indicators, e Brazil, unidas pelo operador boleano AND, da qual limitou-se para estudos publicados de 2000 até 2012. Um total de 14 artigos longitudinais e transversais foram incluídos nessa revisão e os fatores e preditores de risco investigados, tanto na dentição decídua quanto na permanente, foram divididos em componentes biológicos, comportamentais e socioeconômicos. Resultados: assim, foi possivel observar que a cárie está associada a diferentes fatores e preditores de riscos e os mais consolidados são: experiência passada de cárie, defeitos de esmalte, biofilme dental, dieta, escolaridade da mãe e renda. Considerações finais: os fatores e preditores de risco estudados são diversos, e sua identificação é de fundamental relevância para o desenvolvimento de estratégias direcionadas no intuito de diminuir a incidência e a prevalência da cárie.

Palavras-chave: Cárie dentária. Epidemiologia. Prevalência. Incidência. Fatores de risco.

\section{Introdução}

A cárie é considerada um problema de saúde pública devido à sua característica generalizada, ao custo do tratamento e aos efeitos sobre a qualidade de vida da população ${ }^{1}$. Apesar do seu declínio nas últimas décadas, os níveis elevados da doença ainda têm sido identificados em uma minoria de sujeitos, os chamados indivíduos de alto risco-cárie ${ }^{2,3}$. Narvai et al. ${ }^{4}$ (2006) ao analisarem a evolução da experiência de cárie entre escolares brasileiros no período de 1980 a 2003, verificaram através do índice dentes cariados, perdidos ou obturados de (CPOD) que houve um declínio da cárie no período avaliado. Entretanto, foram verificadas diferenças significativas entre as regiões Norte, Nordeste e Centro-Oeste e as regiões Sudeste e Sul. Adicionalmente, os autores desse estudo relatam que a distribuição da cárie está ocorrendo em níveis de desigualdade, cerca de $60 \%$ da doença concentram-se em $20 \%$ da população de escolares. Observa-se a presença de um grupo populacional de alto risco ${ }^{5,6}$.

Dados mais recentes, do SB Brasil 2010, mostram que, aos cinco anos de idade, uma criança brasileira possui CPO médio de 2,43. Comparando-se esse valor com o obtido no SB Brasil 2003, que foi de 2,8 , observa-se que houve uma redução de apenas $13,9 \%$ em sete anos. Comparando os resultados

\footnotetext{
Mestre em Odontologia, Faculdade de Odontologia de Piracicaba (FOP) - Unicamp, Piracicaba-SP, Brasil.

Mestre em Saúde Coletiva, Faculdade de Odontologia de Piracicaba (FOP) - Unicamp, Piracicaba-SP, Brasil.

** Professor livre-docente, Faculdade de Odontologia de Piracicaba (FOP) - Unicamp, Piracicaba-SP, Brasil.

*** Mestre em Odontologia, Faculdade de Odontologia de Piracicaba (FOP) - Unicamp, Piracicaba-SP, Brasil.
} 
do SB Brasil 2003 e 2010 para o índice CPO aos 12 anos, observa-se que o valor passou de 2,78 em 2003 para 2,07 em 2010, correspondendo a uma redução de $26,2 \%$ em sete anos. Desses dentes com experiência de cárie, o predomínio foi do componente cariado, que é responsável por mais de $80 \%$ do índice, mostrando que essas crianças não foram tratadas. Além disso, essa proporção de dentes cariados é sensivelmente maior nas regiões Norte e Nordeste, enquanto a de dentes restaurados é maior nas regiões Sudeste e Sul, o que confirma, ainda nos dias de hoje, a existência de grupos de risco, devido principalmente a fatores de ordem social e à falta de acesso aos sistemas de saúde.

A identificação precoce desses grupos de indivíduos permite que as autoridades de saúde planejem medidas preventivas e utilizem de forma mais eficiente os recursos ${ }^{7}$. Apenas o conceito de que cárie é uma doença multifatorial não permite que sejam aplicadas estratégias para o seu controle ${ }^{8}$. Limitar 0 controle da doença à remoção periódica de biofilme e restrição do consumo de açúcar é uma simplificação biológica do processo, sem considerar determinantes sociais da doença. Então, para que o entendimento global da doença possa ocorrer, é fundamental que estudos sejam conduzidos de forma a identificar os fatores de risco e preditor/indicadores de risco.

Fatores de risco são variáveis biológicas, ambientais e comportamentais que, se presentes, podem aumentar diretamente a ocorrência de determinada doença, ou, se reduzidas ou eliminadas, podem diminuir a ocorrência ${ }^{2,3,9}$. Alguns critérios são necessários para que uma variável seja considerada fator de risco: a presença do fator deve preceder a ocorrência da doença, e deve ter associação estatística e não ser relacionada ao acaso ou atribuída a erro amostral. O risco é expresso pela incidência, que corresponde ao número de casos novos que surgiram em um determinado período de tempo e que, por isso, são confirmados em estudos longitudinais. A força ou a intensidade de progressão ou recuo da doença na população é expressa por meio do Risco Relativo (RR) que é a razão entre duas incidências?

Os preditores de risco estão associados a um risco elevado da doença, mas não faz parte da cadeia causal, ou seja, da etiologia dela ${ }^{3}$. Ao conduzir um estudo transversal, variáveis associadas com a cárie dentária são consideradas como indicadores de risco, que podem ser ou não fatores de risco da doença. O Odds Ratio (OR), ou razão de chances, constitui também uma medida própria de risco, é um dado que estima o risco relativo ${ }^{7}$.

Considerando a cárie um problema de saúde pública que recebe interferência de vários fatores além dos seus agentes etiológicos, os quais são responsáveis pela formação de grupos de polarização, o objetivo deste trabalho foi fazer uma revisão da literatura para verificar quais os fatores e os preditores de risco da cárie em crianças que foram mais estudados no Brasil.

\section{Materiais e método}

Foi realizada uma busca na literatura através do uso da base de dados do PUB MED, utilizando as palavras chaves dental caries, risk indicators, e Brazil, unidas pelo operador boleano AND. Nessa busca inicial foram encontrados 69 artigos, na data 06 de setembro de 2012. Desses artigos resultantes da busca inicial, foram excluídos revisões de literatura, artigos publicados em revistas com classificação Qualis B2 ou abaixo, artigos publicados há mais de dez anos, aqueles que abordavam doenças periodontais, trauma, ou outros tipos de doenças bucais além da cárie dental e aqueles relacionados a pacientes com necessidades especiais e adultos, resultando em uma quantidade final de 14 artigos. Foram incluídos artigos que investigaram os fatores e preditores de risco em ambas as dentições, decídua e permanente. Após a leitura integral desses artigos, foi preenchida uma ficha contendo informações relevantes do trabalho para a revisão de literatura e discussão. Algumas informações relevantes sobre o delineamento e a metodologia dos artigos estão apresentadas na Tabela 1. Os fatores e preditores de risco investigados nos artigos selecionados foram divididos em componentes biológicos, comportamentais e socioeconômicos. 
Tabela 1- Informações sobre delineamento e metodologia de cada artigo

\begin{tabular}{|c|c|c|c|c|c|c|c|}
\hline Referência & Idade & Local & Tipo de estudo & $\mathrm{N}$ & Origem & $\begin{array}{c}\text { Critérios } \\
\text { diagnóstico de } \\
\text { cárie }\end{array}$ & Dentição \\
\hline Santos e Soviero ${ }^{10}(2002)$ & 36 meses & Rio de Janeiro - RJ & Transversal & 80 & Maternidade & $\begin{array}{c}\text { BjØrndal et al. }{ }^{24} \\
\text { (1997) }\end{array}$ & Decídua \\
\hline Ferreira e Mendes ${ }^{11}$ (2005) & 7 a 12 anos & Natal - RN & Transversal & 300 & Escolas públicas & OMS & Ambas \\
\hline Tiano et al..$^{12}(2009)$ & 36 meses & Interior de SP & Transversal & 41 & Creches & OMS & Decídua \\
\hline Tagliaferro et al. ${ }^{13}$ (2006) & 6 a 8 anos & Piracicaba - SP & Longitudinal & 480 & Escolas públicas & OMS & Ambas \\
\hline Kassawara et al. ${ }^{14}$ (2010) & 9 e 10 anos & Piracicaba - SP & Longitudinal & 765 & Escolas públicas & OMS & Permanente \\
\hline Oliveira et al. ${ }^{15}(2006)$ & 0 a 36 meses & João Pessoa - PB & Longitudinal & 256 & Maternidade & OMS & Decídua \\
\hline Targino et al. ${ }^{16}(2011)$ & 24 a 54 meses & João Pessoa - PB & Longitudinal & 224 & Maternidade & OMS & Decídua \\
\hline Peres et al. ${ }^{17}$ (2005) & 1 a 6 anos & Pelotas - SC & Longitudinal & 359 & Maternidade & OMS & Decídua \\
\hline Saraiva et al. ${ }^{18}(2007)$ & 7 a 11 anos & São Luís - MA & Transversal & 2439 & Populacional & OMS & Permanente \\
\hline Cruvinel et al. ${ }^{19}(2010)$ & 5 a 10 anos & Brasilia - DF & Longitudinal & 80 & Maternidade & OMS & Ambas \\
\hline Freire et al..$^{20}(2008)$ & 15 anos & Goiânia - GO & Transversal & 761 & Escolas públicas & OMS & Permanente \\
\hline Feldens et al..$^{21}(2010)$ & 4 anos & São Leopoldo - SP & Longitudinal & 340 & Maternidade & $\begin{array}{c}\text { NIH (Drury et al. } \\
1999)^{25}\end{array}$ & Decídua \\
\hline Ribeiro et. al. ${ }^{22}(2005)$ & 48 meses & João Pessoa - PB & Transversal & 224 & Maternidade & OMS & Decídua \\
\hline Melo et al. ${ }^{23}(2011)$ & $\begin{array}{c}18 \text { a } 36 \text { meses } \\
5 \text { anos }\end{array}$ & Recife - PE & Caso-controle & $\begin{array}{l}1415 \\
605\end{array}$ & USF & OMS & Decídua \\
\hline
\end{tabular}

\section{Revisão de literatura}

Nos artigos incluídos nesta revisão da literatura foi possível identificar quais são os fatores e os preditores de risco de cárie mais comumente identifica- dos. Essas variáveis foram divididas em componentes biológicos, comportamentais e socioeconômicos, que estão expostos na Tabela 2 e serão apresentados a seguir.

Tabela 2 - Tabela comparativa entre os fatores e indicadores de risco estudados em cada artigo

\begin{tabular}{|c|c|c|c|c|c|c|c|c|c|c|c|c|c|c|c|c|c|c|c|c|c|c|c|}
\hline Referência & 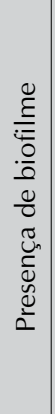 & 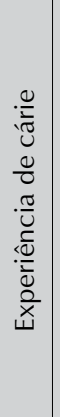 & 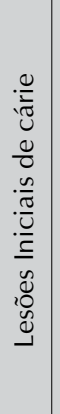 & 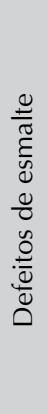 & 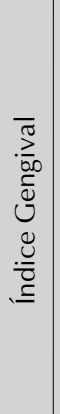 & 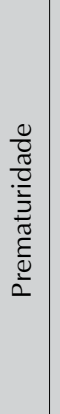 & $\frac{\stackrel{\varpi}{ٍ}}{\frac{\Xi}{<}}$ & $\begin{array}{l}\frac{O}{\bar{d}} \\
\stackrel{\mathbb{E}}{\mathbb{S}}\end{array}$ & $\begin{array}{l}\frac{0}{2} \\
\frac{\pi}{0}\end{array}$ & 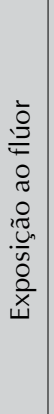 & 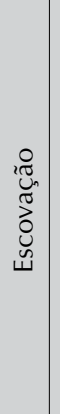 & 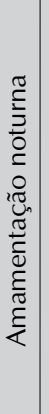 & 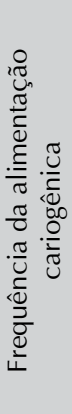 & 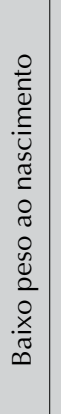 & 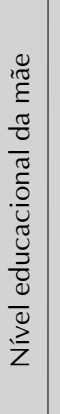 & 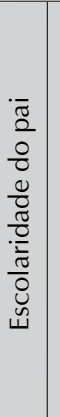 & 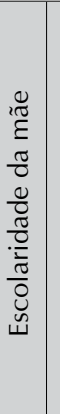 & 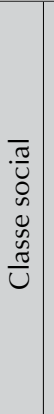 & 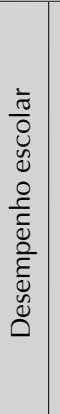 & 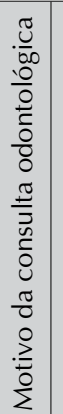 & 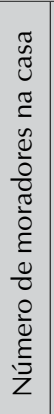 & 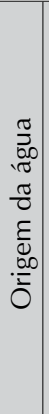 & 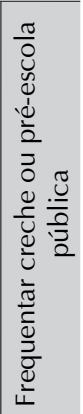 \\
\hline Santos e Soviero ${ }^{10}(2002)$ & + & & & & & & & & & & + & + & & & & & & & & & & & \\
\hline Ferreira Mendes ${ }^{11}(2002)$ & + & - & & & - & & & - & - & & & & & & & & & & & & & & \\
\hline Tiano et al. ${ }^{12}(2009)$ & & + & & & & & & & & & + & + & & & & & & & & & & & \\
\hline Tagliaferro et al. ${ }^{13}$ (2006) & & + & & & & & & & & & & & & & + & & & & & & & & \\
\hline Kassawara et al. ${ }^{14}(2012)$ & & + & + & & & & & & & & & & & & + & & & & & & & & \\
\hline Oliveira et al. ${ }^{15}$ (2006) & & & & + & & & & & & & + & + & & & & & & & & & & & \\
\hline Targino et al. ${ }^{16}(2011)$ & & & & + & & & & & & & & & & & & & & & & & & & \\
\hline Peres et al. ${ }^{17}$ (2005) & & & & & & & & & & & + & & + & + & & + & & + & & & & & \\
\hline Saraiva et al. ${ }^{18}(2007)$ & & & & & & & & & & & & & & - & & & & & & & & & \\
\hline Cruvinel et al. ${ }^{19}(2010)$ & & & & & & - & & & & & & & & & & & & & & & & & \\
\hline Freire et al..$^{20}(2008)$ & & & & & & & + & & & & & & & & & & & + & + & & & & \\
\hline Feldens et al. ${ }^{21}$ (2010) & & & & & & & & - & - & & & + & + & & & & + & & & & & & \\
\hline Ribeiro et al. ${ }^{22}$ (2005) & & & & + & & & & + & & + & + & + & + & & & & & & & & & & \\
\hline Melo et al. ${ }^{23}(2011)$ & & & & & & & & & & & & & + & & & & + & & & + & + & + & \\
\hline
\end{tabular}

* Simbolo "+" indica que a variável apresentou correlação estatística com o CPOD $(p<0,05)$. Simbolo "-” indica que a variável foi estudada pelo estudo, porém não apresentou correlação estatística; Símbolos em negrito indicam que o fatores de risco (estudos longitudinais) e os demais expressam preditores de risco. 


\section{Componentes biológicos}

Os fatores biológicos estudados podem ser divididos em bucais, ou de ordem geral. Dentre os fatores bucais, os mais estudados foram lesões de mancha branca, experiência prévia de cárie, defeitos de esmalte, experiência passada de cárie, presença de biofilme, entre outros e de ordem geral, destacaram-se prematuridade, peso e estatura.

A presença significativa de biofilme dental e sua associação com a cárie dentária $(\mathrm{p}<0,001)$ foi verificada em 2002, através de um estudo cujo objetivo foi avaliar a prevalência de cárie e os fatores de risco em crianças cadastradas no Ambulatório de Pediatria do Hospital Universitário Pedro Ernesto (HUPE-UERJ) com idade até 36 meses $^{10}$. Em outro estudo transversal ${ }^{11}$, porém, com crianças de sete a 12 anos de idade, estudantes de escolas públicas da cidade de Natal- RN, que possuíam lesão de mancha branca ativa na vestibular do incisivo superior foi também verificada a associação da presença de biofilme com a prevalência de cárie. Os autores verificaram que o índice de placa mostrou correlação com o número de lesões $(\mathrm{p}=0,006)$, considerando que em $85 \%$ dos sítios com lesão de mancha branca, havia presença de placa. Além de presença de biofilme, os autores avaliaram outras variáveis: índice de cárie, índice gengival, gênero e idade, porém para essas variáveis não se verificou correlação estatística com o índice de cárie. Ainda no que diz respeito às lesões de mancha branca, Tiano et al. ${ }^{12}$ (2009), ao avaliarem a prevalência de mancha branca associada à qualidade de higiene oral em crianças residentes de cidade do interior de São Paulo com idade de até 36 meses, verificaram que a duração do hábito de mamar durante a noite (até mais de 12 meses), a idade de início da prática de higiene oral, e a presença de lesões cavitadas estiveram associados com a presença de lesões iniciais em esmalte.

Em um estudo longitudinal, Tagliaferro et al. ${ }^{13}$ (2006) tiveram como objetivo identificar se fatores clínicos, comportamentais, demográficos e socioeconômicos podem ser considerados preditores da cárie dentária na dentição permanente de crianças com idade entre seis e oito anos de três diferentes escolas de Piracicaba SP. Dentre os fatores biológicos, o risco de cárie mostrou-se associado à experiência de cárie, além do nível educacional da mãe (componente socioeconômico). Outro estudo mais recente, também realizado na cidade de Piracicaba, investigou como a experiência de cárie, em lesões iniciais e os estágios cavitados, predizem o incremento de cárie em dentes permanentes em um período de dois anos. Os autores concluíram que houve associação estatística entre lesão inicial e incremento de cárie na faixa etária de nove a dez anos e entre experiência de cárie, tanto em dentes decíduos quanto em permanentes e o incremento de cárie ${ }^{14}$.

Com relação à avaliação da influência dos defeitos de esmalte no desenvolvimento da cárie, um estudo longitudinal, do nascimento aos 36 meses, em crianças de baixo nível socioeconômico, residentes em João Pessoa - PB, avaliou essa associação ${ }^{15}$. Os resultados desse estudo demonstraram que houve associação entre cárie e defeitos de esmalte $(\mathrm{p}=0,0001)$. A chance (OR) decresce com a idade: 29,4 para os 18 meses, 27,1 para os 24 meses e 16,37 para os 30 meses. Entretanto, apenas 0,9\% dos dentes sem defeitos de esmalte desenvolveram cárie. A análise multifatorial mostrou que defeitos de esmalte foi fator de risco para o desenvolvimento de cárie aos 18, 24, 30 e até aos 36 meses $(\mathrm{p}<0,05)$. Em estudo publicado mais recentemente, verificou-se que defeitos na estrutura dental do esmalte constituem fator predisponente à cárie de erupção preco$\mathrm{ce}^{16}$. Nesse estudo, também realizado na cidade de João Pessoa - PB, foram avaliadas 224 crianças a cada seis meses, desde os 24 meses até os 54 meses de idade. Foi verificado que uma criança que apresentasse defeito no esmalte tinha duas vezes mais chance de desenvolver cárie, em que a hipoplasia do esmalte foi o tipo mais frequente de defeito.

Dentre os fatores biológicos de ordem geral, a prematuridade foi estudada como fator de risco ou predisponente da cárie dentária. Em um estudo longitudinal foi avaliada a associação entre condições biológicas no primeiro ano de vida, além das sociais, com o índice de cárie em crianças com seis anos de idade divididas em grupos: CPOD $\leq 1$ e CPOD $\geq 4$. Observou-se que as crianças abaixo do peso aos 12 meses apresentaram chance (OR) 4,1 vezes maior de apresentar alto índice de cárie ${ }^{17}$. Entretanto, Saraiva et al. ${ }^{18}$ (2007) também tiveram como objetivo avaliar a associação entre baixo peso ao nascimento, segundo relato da mãe em questionário, e CPOD aos sete e 11 anos de idade, porém, os autores não encontraram correlação estatística. Esses resultados também corroboram o estudo transversal realizado com 80 crianças que nasceram prematuras no Hospital Regional da Asa Sul (HRAS) de Brasília, no qual os autores concluíram que a prematuridade não é um fator predisponente para cárie ${ }^{19}$.

Outro fator avaliado como possível componente biológico de ordem geral foi a estatura das crianças. A hipótese de que adolescentes mais altos possuiriam menor nível de experiência de cárie foi testada e os resultados de um estudo transversal realizado em Goiás com 664 adolescentes de 15 anos de escolas da área urbana (50\% privadas e 50\% públicas) verificou a associação entre altura, classe social e desempenho escolar com o índice de cárie (CPOD e CPOS ${ }^{20}$.

\section{Componentes comportamentais}

No que se refere aos componentes comportamentais, alguns estudos buscam investigar a associação estatística entre cárie e hábitos dietéticos (frequência de consumo de alimentos cariogênicos, amamentação, idade de introdução do açúcar e hi- 
giene (frequência de escovação, uso de dentifrício fluoretado, entre outros).

Pesquisando sobre a associação entre cárie e dieta, um estudo longitudinal realizado em São Leopoldo - SP investigou se os hábitos alimentares, no primeiro ano de vida, estavam associados com a cárie severa e a precoce em crianças de quatro anos de idade e verificou-se que a duração da alimentação, a frequência, o uso da mamadeira noturna, especialmente para outros líquidos que não o leite, a presença de alta densidade de açúcar e de lipídios, além de fatores socioeconômicos estão associados com o incremento de cárie. Entretanto, os autores verificaram que não houve associação para idade, gênero e idade de introdução do açúcar ${ }^{21}$.

Ainda com relação aos hábitos alimentares e de higiene, Peres et al. ${ }^{17}$ (2005), mostraram que crianças que consumiam alimentos cariogênicos pelo menos uma vez ao dia aos seis anos tinham chance 2,3 vezes maior de ter alto índice de cárie, e as que escovavam os dentes menos de uma vez ao dia, tinham 3,1 vezes mais chance. Porém, um estudo longitudinal, realizado por Ribeiro et al..$^{22}$ (2005), em João Pessoa - PB, teve como objetivo verificar a prevalência de cárie precoce em crianças com 48 meses de idade de baixo nível socioeconômico. Foi verificada uma correlação entre o gênero, os hábitos alimentares, a higiene, a exposição ao flúor e a presença de defeitos de esmalte, porém os fatores comportamentais de risco (dieta, higiene, uso de flúor, amamentação natural e artificial) não apresentaram relação estatística com a doença $(p>0,05)$. Essa conclusão corrobora o estudo realizado por Santos e Soviero ${ }^{10}$ (2002), citado no tópico anterior (Fatores biológicos), que trabalhou com crianças de até 36 meses de idade cadastradas no Ambulatório de Pediatria do Hospital Universitário Pedro Ernesto (HUPE-UERJ), onde foi avaliada a associação entre a prevalência de cárie e frequência de higiene, hábito de amamentação noturna e dieta cariogênica durante o dia, não ocorrendo, porém, associação estatisticamente significativa. Já outros autores, como Oliveira et al. ${ }^{15}$ (2006) ao avaliarem fatores de risco de cárie em crianças de João Pessoa - PB do nascimento aos 36 meses, observaram que além dos defeitos de esmalte, também apresentados no tópico anterior, os hábitos de higiene aos 18 meses e a amamentação noturna aos 18 e aos 24 meses foram fatores de risco para o desenvolvimento de cárie até os 36 meses $(\mathrm{p}<0,05)$.

\section{Componentes socioeconômicos}

Dentre os diversos componentes socioeconômicos, destacamos: escolaridade dos pais, renda familiar, dados da moradia, abastecimento de água, dentre outros. A escolaridade dos pais é um fator que tem sido investigado nos grupos de risco. No estudo de Peres et al. ${ }^{17}$ (2005), as variáveis socioeconômicas que apresentaram associação significante com o alto índice de cárie foram: escolaridade do pai e da mãe que trabalharam durante a gravidez. Além dessas, observou-se que crianças cujos pais estavam desempregados quando no nascimento, apresentaram uma chance 7,7 vezes maior de ter alto índice de cárie.

No mesmo estudo longitudinal conduzido por Tagliaferro et al. ${ }^{13}$ (2006), supracitado em "Componentes biológicos", foram avaliados os fatores socioeconômicos e foi observado que o nível de escolaridade da mãe ( $\leq 8$ anos de estudo) pode ser considerado um preditor da cárie na dentição permanente de crianças com idade entre seis e oito anos de Piracicaba-SP $(\mathrm{p}=0,03)$. Resultados que corroboram com um estudo longitudinal realizado em São Leopoldo - SP, em que foi encontrada uma associação estatística entre cárie e escolaridade na mãe $(\mathrm{p}=0,007)^{21}$.

Em outro estudo de caso controle mais recente ${ }^{23}$, realizado com crianças nas faixas etárias entre $18 \mathrm{e}$ 36 meses e cinco anos, cadastradas nas unidades de saúde família (USF) da ESF de dois Distritos Sanitários (DS) do Município do Recife, em que se dividiu o grupo de estudo em caso (ceo-d $\geq 1$ ) e controle (ceo-d $=0$ ). Foi observado que: baixa escolaridade materna $(<8$ anos de estudo), moradia em domicílios com seis ou mais moradores, mais de três pessoas por quarto e tempo de moradia na área de três ou mais anos estiveram estatisticamente associados à experiência de cárie dentária nos grupos etários investigados. Já para a faixa etária entre 18-36 meses, o fato de a origem da água do domicílio ser de poço/nascente apresentou-se negativamente associado a um ceo-d $\geq 1$. Em crianças de cinco anos, mães que não trabalham fora se constituíram em fator associado para a cárie no grupo entre 18-36 meses $(p=0,0239)$. No que se refere a morar com três ou mais irmãos em ambas as idades, ou com dois irmãos, e a ser primeiro no grupo de cinco anos, foram fatores que se associaram à cárie. Não frequentar creches constituiu um fator associado à ocorrência de cárie aos 18-36 meses. Frequentar creche ou pré-escola pública mostrou associação para a faixa etária de 18-36 meses e para a idade de cinco anos. Finalmente, frequentar creche ou pré-escola filantrópica associou-se apenas com a idade de cinco anos.

\section{Discussão}

A experiência passada de cárie na dentição decídua é um fator preditor de cárie para a dentição permanente $^{13}$. Além da experiência passada de cárie, lesões iniciais predizem o incremento de cárie em dentes permanentes em um período de dois $\operatorname{anos}^{14}$. A presença de biofilme e lesões de mancha branca foi identificada como preditor de risco para a cárie dental, tanto em crianças joven $\mathrm{s}^{10}$, quanto numa faixa etária mais avançada ${ }^{11}$. 
Os defeitos de esmalte também foram um fator associado com cárie dental, considerando que num estudo longitudinal, Oliveira et al. ${ }^{15}$ (2006), concluíram que do nascimento aos 18,24, 30 e até os 36 meses a chance de desenvolver cárie é maior em crianças que apresentam defeitos de esmalte, porém, o OR decresce com a idade, o que pode sugerir que com a idade, outros fatores passam a exercer maior influência nessa relação. Essa condição foi demonstrada também em outro estudo conduzido na mesma população, porém dos 24 aos 54 meses de idade, no qual, ao calcular-se o Risco Relativo (RR), concluiu-se que o risco de desenvolver cárie é cerca de duas vezes maior aos 54 meses para crianças que tinham defeitos de esmalte ${ }^{16}$.

Já a prematuridade, avaliada por alguns autores não foi identificada como um preditor de risco para cárie. Porém, isso pode ter acontecido devido ao desenho do estudo, transversal, que se baseou nos registros de prematuridade da maternidade, mas avaliou crianças já em idade mais avançada, cinco e dez anos de idade, e não de forma longitudinal desde os primeiros anos de vida ${ }^{19}$. Pois, em um estudo longitudinal realizado anteriormente ${ }^{17}$, foi observado que crianças com baixo peso aos 12 meses, sem especificação de prematuridade, apresentaram maior risco de ter alto índice de cárie. Resultados divergentes foram obtidos por Saraiva et al. ${ }^{18}$ (2007), pois não encontraram associação estatística entre peso no nascimento e CPOD na faixa etária dos sete aos 11 anos de idade, porém os dados de peso ao nascimento desse estudo foram obtidos por meio de questionário respondido pelo responsável no momento do estudo, enquanto no desenho de estudo realizado por Peres et al. ${ }^{15}$ (2005), o peso foi obtido por pesagem da criança, o que seria mais confiável.

Num estudo longitudinal, foi observado que dentre os componentes comportamentais, os hábitos alimentares do primeiro ano de vida - duração da alimentação, frequência, uso da mamadeira noturna para outros líquidos que não o leite, presença de alta densidade de açúcar e de lipídios - estão associados ao incremento de cáries obtido aos quatro anos de idade. Porém, a idade de introdução do açúcar não obteve associação estatística ${ }^{21}$. Oliveira et al..$^{15}$ (2006) também observaram que a amamentação noturna numa idade mais avançada, aos $18 \mathrm{e}$ aos 24 meses constitui fator de risco para o desenvolvimento de cárie até os 36 meses de idade, além dos hábitos de higiene aos 18 meses.

Entretanto, no estudo conduzido por Ribeiro et al. $^{22}$ (2005), não foi observada uma associação significativa entre as variáveis comportamentais analisadas (dieta, higiene, uso de flúor, amamentação natural e artificial) e a cárie precoce da infância (até os 48 meses). Também não houve associação estatística entre prevalência de cárie e frequência de higiene, hábito de amamentação noturna e dieta cariogênica durante o dia, em crianças de até 36 meses de idade, no estudo de Santos e Soviero ${ }^{10}$ (2002).
No mesmo estudo, houve correlação negativa entre uso de dentifrício fluoretado e índice de cáries, porém não foi calculado o $\mathrm{OR}^{10}$.

No que diz respeito aos fatores socioeconômicos, o nível educacional da mãe ( $\leq 8$ anos de estudo) pode ser considerado um preditor da carie dentária na dentição permanente, resultando em um risco 1,88 vezes maior, quando observado em crianças dos seis aos oito anos ${ }^{13}, 1,5$ vezes, desde o nascimento até aos quatro anos ${ }^{21}$, e 3,91 para crianças mais jovens, dos 18 aos 36 meses $^{23}$. A baixa escolaridade do pai apresentou associação com o alto índice de cárie apenas em Peres et al. ${ }^{17}$ (2005). Além dos domicílios com seis ou mais moradores, mais de três pessoas por quarto, com um tempo de moradia na área de três anos ou mais, o fato de não frequentar creches, ou frequentar creche ou pré-escola pública também constituiu fatores associados à ocorrência de cárie aos 18-36 meses. Já o fato de a origem da água do domicílio ser de poço/nascente apresentou-se negativamente associado a um ceo-d $\geq 1^{23}$.

Embora esse estudo possua limitações por ser uma revisão de literatura convencional e não sistemática, e que teve como fonte de busca uma única base de dados, é possível verificar os diferentes fatores e preditores de riscos associados à cárie dentária.

\section{Conclusão}

A cárie dentária é uma doença que tem apresentado um declínio nos últimos anos, porém existem grupos populacionais que apresentam alto risco de desenvolvimento dessa doença. Logo, o estudo dos fatores e indicadores de risco é relevante para o desenvolvimento de estratégias que possam diminuir a incidência de cárie. Dentre tais fatores, os mais consolidados na literatura são: experiência passada de cárie, defeitos de esmalte, biofilme dental, dieta, escolaridade da mãe e renda.

\section{Abstract}

Currently, the prevalence of dental caries is located in a portion of the population, characterizing the polarization. The identification of these risk groups allows preventive measures, both community and individual, should be directed to these individuals, resulting in more efficient use of resources. Objective: identify factors and predictors of caries risk studied in Brazil in a review of the literature. Materials and method: a search was made in the PUBMED using the terms "dental caries AND risk indicators AND Brazil", from witch gets studies published from 2000 to 2012. A total of 14 articles, longitudinal and transversal, were included in this review and the risk factors and predictors investigated, either in deciduous either in permanent dentition, were divided into biological, behavioral and socioeconomic factors. Results: It was observed that the decay is associated with different risk factors and predictors and the most consolidated are: past experience of caries, enam- 
el defects, dental biofilm, diet, mother's education and income. Final considerations: The factors and predictors of caries risk studied were diverses, and the identification of these factors in risk groups is of fundamental importance for the development of targeted strategies in order to reduce the incidence and prevalence of caries.

Keywords: Dental caries. Epidemiology. Prevalence. Incidence. Risk factors.

\section{Referências}

1. Hausen H. Caries prediction: state of the art. Community Dent Oral Epidemiol 1997; 25:87-96.

2. Tagliaferro EPS, Ambrosano GMB, Meneghim MC, Pereira AC. Risk indicators and risk predictors of dental caries in schoolchildren. J Appl Oral Sci 2008; 16(6):408-13.

3. Tagliaferro EPS, Pardi V, Ambrosano GMB, Meneghim MC, Pereira AC. An overview of caries risk assessment in 0-18 yars-olds over the last ten years (1997-2007). Braz J Oral Sci 2008; 27(7):1682-90.

4. Narvai PC, Frazão P, Roncalli AG, Antunes JLF. Cárie dentária no Brasil: declínio, iniquidade e exclusão social. Rev Panam Salud Publica 2006; 19(6):385-93.

5. Poulsen S, Scheutz F. Dental caries in Danish children and adolescents 1988-1997. Community Dent Health 1999; 16(3):166-70.

6. Pereira HP, Cossta VR, Antunes LAA, Perez ME, Costa R. Caries disease as caries predictors in deciduous and permanent teeth. Arq em Odontol 2009; 45:67-72.

7. Pereira AC. Tratado de Saúde Coletiva em Odontologia. 2. ed. Nova Odessa Napoleão; 2009

8. Tenuta LMA, Chedid SJ, Cury JA. Uso de fluoretos em Odontopediatria - Mitos e Evidências. In: Maia LC, Primo LG, Iazzetti GJ. Odontologia Integrada na infância. 1. ed. Santos; 2012. p.154.

9. Beck JD. Risk revisited. Community Dent Oral Epidemiol 1998; 26:220-5.

10. Santos AP, Soviero VM. Caries prevalence and risk factors among children aged 0 to 36 months. Pesq Odontol Bras 2002; 16(3):203-8.

11. Ferreira MA, Mendes NS. Factors associated with active white enamel lesions. Int J Paediatr Dent 2005; 15(5):32734 .

12. Tiano AV, Moimaz SA, Saliba O, Garbin CA. Prevalence of enamel white spots and risk factors in children up to 36 months old. Braz Oral Res 2009; 23(2):215-22.

13. Tagliaferro EPS, Pereira AC, Meneghim MC, Ambrosano GMB. Assessment of dental caries predictors in a seven-year longitudinal study. J Public Health Dent 2006; 66:169-73.

14. Kassawara AB, Tagliaferro EP, Cortelazzi KL, Ambrosano GM, Assaf AV, Meneghim M de C, et al. Epidemiological assessment of predictors of caries increment in 7-10- year-olds: a 2-year cohort study. J Appl Oral Sci 2010; 18(2):116-20.

15. Oliveira AF, Chaves AM, Rosenblatt A. The influence of enamel defects on the development of early childhood caries in a population with low socioeconomic status: a longitudinal study. Caries Res 2006; 40(4):296-302.

16. Targino AG, Rosenblatt A, Oliveira AF, Chaves AM, Santos VE. The relationship of enamel defects and caries: a cohort study. Oral Dis 2011; 17(4):420-6.

17. Peres MA, de Oliveira Latorre M, Sheiham A, Peres KG, Barros FC, Hernandez PG, et al. Social and biological early life influences on severity of dental caries in children aged 6 years. Community Dent Oral Epidemiol 2005; 33(1):53-63.
18. Saraiva MC, Chiga S, Bettiol H, Silva AA, Barbieri MA. Is low birthweight associated with dental caries in permanent dentition? Paediatr Perinat Epidemiol 2007; 21(1):49-56.

19. Cruvinel VR, Gravina DB, Azevedo TD, Bezerra AC, Toledo OA. Prevalence of dental caries and caries-related risk factors in premature and term children. Braz Oral Res 2010; 24(3):329-35.

20. Freire MC, Sheiham A, Netuveli G. Relationship between height and dental caries in adolescents. Caries Res 2008; 42(2):134-40.

21. Feldens CA, Giugliani ER, Vigo Á, Vítolo MR. Early feeding practices and severe early childhood caries in four-year-old children from southern Brazil: a birth cohort study. Caries Res 2010; 44(5):445-52.

22. Ribeiro AG, de Oliveira AF, Rosenblatt A. Early childhood caries: prevalence and risk factors in 4-year-old preschoolers in João Pessoa, Paraíba, Brasil. Cad Saúde Pública $2005 ; 21(6): 1695-700$

23. Melo MM, Souza WV, Lima ML, Braga C. Factors associated with dental caries in preschoolers in Recife, Pernambuco State, Brazil. Cad Saúde Publica 2011; 27(3):471-85.

Endereço para correspondência:

Lenita Marangoni Lopes

Av. Limeira, 901

13414-903 Piracicaba-SP

Fone: (11) 21065290

E-mail: lenita_m_|@hotmail.com

Recebido: 29/08/2013. Aceito: 07/08/2014. 